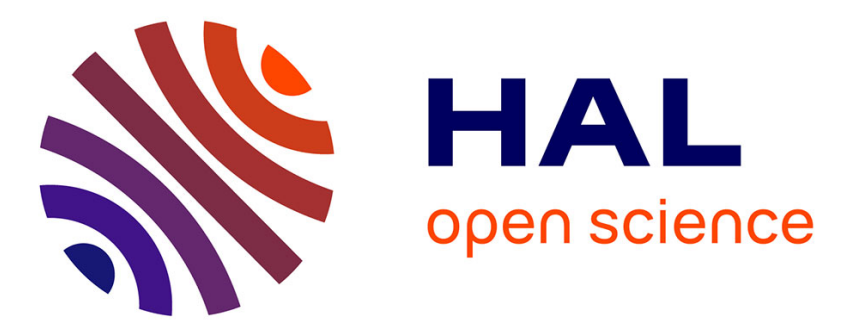

\title{
Une méthode impulsionnelle de mesure de la durée de vie des porteurs injectés dans la zone centrale d'une structure pin
}

\author{
J. Barrau, L. Bailon, J.-C. Brabant, M. Brousseau
}

\section{To cite this version:}

J. Barrau, L. Bailon, J.-C. Brabant, M. Brousseau. Une méthode impulsionnelle de mesure de la durée de vie des porteurs injectés dans la zone centrale d'une structure pin. Revue de Physique Appliquée, 1971, 6 (1), pp.19-22. 10.1051/rphysap:019710060101900 . jpa-00243497

\section{HAL Id: jpa-00243497 https://hal.science/jpa-00243497}

Submitted on 1 Jan 1971

HAL is a multi-disciplinary open access archive for the deposit and dissemination of scientific research documents, whether they are published or not. The documents may come from teaching and research institutions in France or abroad, or from public or private research centers.
L'archive ouverte pluridisciplinaire HAL, est destinée au dépôt et à la diffusion de documents scientifiques de niveau recherche, publiés ou non, émanant des établissements d'enseignement et de recherche français ou étrangers, des laboratoires publics ou privés. 


\title{
UNE MÉTHODE IMPULSIONNELLE \\ DE MESURE DE LA DURÉE DE VIE \\ DES PORTEURS INJECTÉS DANS LA ZONE CENTRALE D'UNE STRUCTURE PIN
}

\author{
J. BARRAU, L. BAILON, J.-C. BRABANT et M. BROUSSEAU \\ Laboratoire de Physique des Solides, associé au C. N. R. S. \\ Faculté des Sciences et I. N. S. A. \\ 118, route de Narbonne, 31, Toulouse (04)
}

(Reçu le 3 octobre 1970)

\begin{abstract}
Résumé. - Les méthodes usuelles [1 à 7] de mesure de la durée de vie des porteurs dans les diodes PIN permettent la détermination d'une durée de vie effective correspondant à la recombinaison totale dans la structure [7] : zone centrale (I) et extrémités fortement dopées $\left(\mathrm{P}^{+}\right.$et $\left.\mathrm{N}^{+}\right)$.

Nous proposons une méthode impulsionnelle, de mise en œuvre facile dans le cas de durées de vie longues $(>0,1 \mu \mathrm{s})$; nous l'interprétons comme une mesure de la durée de vie en volume dans la zone centrale.

Abstract. - Usual methods for carrier-lifetime measurements in PIN structures give effective carrier-lifetimes corresponding to recombinations processes which occur in the whole structure (middle region and hightly-doped ends $\mathrm{P}^{+}$and $\mathrm{N}^{+}$).

We propose a pulse-method for measuring carrier-lifetime in the bulk of the middle region.
\end{abstract}

I. Introduction. - La durée de vie effective des porteurs injectés dans une diode PIN que l'on obtient par les méthodes usuelles [1 à 7] est un paramètre technologique important qui rend compte globalement de phénomènes de recombinaison pouvant être différents et intervenant aussi bien dans la zone centrale que dans les extrémités fortement dopées. L'intérêt d'une méthode de mesure de durée de vie des porteurs qui permettrait de caractériser la recombinaison dans une zone précise de la structure (zone I) serait :

- d'atteindre un paramètre ayant une signification physique précise (durée de vie en volume dans le matériau homogène que constitue la zone centrale) ;

- de permettre une meilleure interprétation des mécanismes de conduction qui déterminent la caractéristique de fonctionnement direct.

Nous avons déjà présenté une méthode d'atténuation hyperfréquence par les porteurs injectés interprétée effectivement comme une méthode de mesure de la durée de vie en volume dans la zone centrale [8,9]. Elle est particulièrement avantageuse pour mesurer des durées de vie brèves $(<0,1 \mu \mathrm{s})$ mais cela n'est pas limitatif.

Son originalité par rapport aux techniques classiques est étudiée actuellement au Laboratoire.

La méthode impulsionnelle que nous présentons ici offre l'avantage d'une mise en œuvre particulièrement simple ; elle conduit à des mesures très précises pour les durées de vies plutôt longues $(>0,1 \mu \mathrm{s})$.

II. Analyse de la réponse transitoire d'une structure PIN. - La tension aux bornes d'une structure $\mathbf{P}^{+}$ $\mathrm{IN}^{+}$parcourue par un courant direct peut être décomposée en 3 termes :

- Les tensions de jonctions $\mathrm{P}^{+} \mathrm{I}$ et $\mathrm{IN}^{+}$notées $V_{j}$;

- Les chutes de tensions dans les zones dopées (zd) :

$$
-\int_{\mathbf{z d}} E(x) \mathrm{d} x \text {. }
$$

- La chute de tension dans la zone centrale (zc) ;

$$
-\int_{z c} E(x) \mathrm{d} x \text {. }
$$

Le champ électrique peut s'exprimer en fonction de la densité de courant total selon :

$$
E(x)=\frac{J-J_{\mathrm{diff}}}{\sigma(x)}
$$

où

$$
\sigma(x)=\mathrm{e} n(x) \mu_{n}+\mathrm{e} p(x) \mu_{p}
$$

et

$$
J_{\mathrm{diff}}=\mathrm{e} D_{n} \frac{\mathrm{d}}{\mathrm{d} x} n(x)-\mathrm{e} D_{p} \frac{\mathrm{d}}{\mathrm{d} x} p(x)
$$

sont respectivement la conductivité et le courant de 
diffusion au point $x$. La tension aux bornes de la structure peut donc s'écrire :

$$
V=V_{j}-\int_{\mathrm{zd}} \frac{J-J_{\mathrm{diff}}}{\sigma(x)} \mathrm{d} x-\int_{\mathrm{zc}} \frac{J-J_{\mathrm{diff}}}{\sigma(x)} \mathrm{d} x .
$$

Supposons que le courant stationnaire appliqué soit supprimé à l'instant $t_{0}$ : la tension subit un changement brusque (phase I ; Fig. 1) suivi d'une décroissance lente (phase II puis III ; Fig. 1).

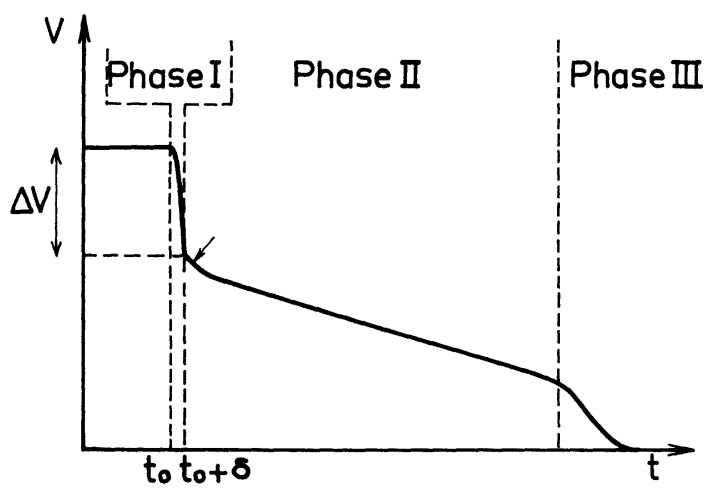

Fig. 1. - Tension aux bornes de la diode en circuit ouvert.

Phase T. - Supposons que le courant appliqué à la structure soit donné par une source de courant dont le temps de descente $\delta$ est plus long que le temps de relaxation diélectrique local en régime d'injection $(\theta=\varepsilon / \sigma)$ et plus court que la durée de vie des porteurs $\tau(\theta \ll \delta \ll \tau)$. Ceci est réalisable le plus souvent sans difficulté en raison des ordres de grandeurs respectifs de $\theta$ et $\tau$ (typiquement $\theta<10^{-9}$ s et $\tau>10^{-7} \mathrm{~s}$ ).

Dans ces conditions :

a. - Le courant de déplacement est négligeable devant le courant total tout au long de la phase I, ce qui entraîne que la tension aux bornes de la structure est donnée à chaque instant par une expression du type (1).

b. - La répartition spatiale de la densité de porteurs reste pratiquement invariable au cours de la phase I, ce qui entraîne l'invariance de $V_{j}$ et des intégrales du type $\int \frac{J_{\text {diff }}}{\sigma(x)} \mathrm{d} x$ dans l'expression (1).

Le point $a$ est établi clairement par Yu. K. Barsukov [10] : cet auteur montre que pour un milieu de conductivité $\sigma$ le courant de déplacement est négligeable devant le courant total pourvu que la vitesse de changement du courant au cours d'une phase transitoire obéisse à la condition :

$$
\left|\frac{I}{J-J_{0}} \frac{\partial\left(J-J_{0}\right)}{\partial t}\right| \ll \frac{\sigma}{\varepsilon}
$$

qui se traduit pratiquement par $\delta \gg \theta$. $\left(J_{0}\right.$ représente le courant stationnaire et $J$ le courant à un instant quelconque de la phase transitoire.)

Le point $b$ est la conséquence des deux observations suivantes : d'une part la condition $\delta \ll \tau$ assure que les densités spatiales d'électrons et de trous n'ont pas changé notablement par recombinaison; d'autre part la distribution spatiale des densités de porteurs n'a pu changer sensiblement du fait de la relaxation des charges d'espace liée au changement du champ électrique : en effet, dans toute la structure la densité de charge d'espace est petite devant la densité de porteurs en régime de double injection [11] (Hypothèse de la quasi-neutralité électrique).

En conséquence, la tension aux bornes de la structure à la fin de la phase I, c'est-à-dire lorsque le courant délivré par la source est devenu nul est donné par (1) lorsque l'on y fait $J=0$, les autres termes restant numériquement inchangés. Le saut de tension $\Delta V$ correspondant (Fig. 1) se déduit par différence :

$$
\Delta V=J \int_{\mathrm{zd}} \frac{1}{\sigma(x)} \mathrm{d} x+J \int_{\mathrm{zc}} \frac{1}{\sigma(x)} \mathrm{d} x
$$

relation dans laquelle $J$ et $\sigma(x)$ sont les valeurs du courant total et de la conductivité locale pendant le régime stationnaire d'injection. Ce saut de tension doit donc être interprété comme la chute ohmique totale dans la structure au cours du régime stationnaire d'injection.

Phase II. - Cette région est caractérisée par une décroissance relativement lente de la tension en fonction du temps. Plusieurs auteurs [4, 5, 6, 7] ont étudié cette phase transitoire et en ont déduit une méthode de mesure de la durée de vie des porteurs injectés dont nous nous bornerons à souligner les caractères importants.

Dans cette phase II, l'équation (1) peut s'écrire :

$$
V=V_{j}(t)+\int_{\mathrm{zd}} \frac{J_{\mathrm{diff}}}{\sigma(x)} \mathrm{d} x+\int_{\mathrm{zc}} \frac{J_{\mathrm{diff}}}{\sigma(x)} \mathrm{d} x .
$$

La tension $V_{j}(t)$ est reliée à la densité de porteurs $n_{j}(t)$ près d'une jonction par la relation [5] :

$$
\frac{n_{j}}{\mathrm{~d} n_{j} / \mathrm{d} t}=\frac{2 k T}{q} \frac{1}{\mathrm{~d} V_{j} / \mathrm{d} t}
$$

(modèle d'injection symétrique).

Donc, si nous omettons les deux termes tenant compte de la diffusion $(*)$, la variation de la tension au cours $\mathrm{du}$ temps (qui n'est plus qu'une tension de jonction) est reliée aux variations des densités de porteurs aux contacts $\mathrm{P}^{+} \mathrm{I}$ et $\mathrm{IN}^{+}$. On comprend donc que la «durée de vie » déduite de la pente de la tension aux bornes de la diode en circuit ouvert dépendra fortement $a$ ) des processus de recombinaisons dans les zones fortement dopées, $b$ ) de la diffusion de porteurs des contacts vers le centre de la structure. En ce qui concerne le point $a$ on voit en effet que l'adoption du modèle de Shockley pour décrire l'évolution de la tension de jonction en régime transitoire implique

(*) Ces termes sont généralement petits ; cependant le premier prend de l'importance à très forte injection. 
l'existence d'un transfert de charges de la zone centrale vers les zones dégénérées. La durée de vie ainsi mesurée doit être interprétée comme une durée de vie effective [6] particulièrement sensible aux processus de recombinaisons qui prédominent au voisinage des jonctions. En ce qui concerne le point $b$, l'équation de continuité des trous [12]:

$$
\frac{\partial \Delta p}{\partial t}=\frac{-\Delta p}{\tau}+D^{*} \frac{\partial^{2} \Delta p}{\partial x^{2}}
$$

montre que la densité locale d'électron et de trou varie à la fois du fait de la recombinaison et de la diffusion ; le gradient important de densité de porteurs près des jonctions est certainement responsable d'une décroissance de la tension de jonction par l'effet de la diffusion des porteurs vers le centre. En particulier, c'est là probablement la raison de la décroissance rapide de la tension que l'on observe au tout début de la phase II (flèche Fig. 1).

III. Méthode de la double impulsion. - $a$. PRINCIPE. - Si nous appliquons à la structure $\mathrm{P}^{+} \mathrm{IN}^{+}$ deux impulsions de courant d'égales intensités $J_{0}$ et séparées par un temps $\Delta t=t_{1}-t_{0}$ la tension aux bornes de la diode présente, à la fin de la première impulsion (instant $t_{0}$ ) une variation brutale de tension $\Delta V_{0}$ suivie d'une décroissance lente déjà examinée. A l'instant $t_{1}$ il se produit un nouveau changement brusque $\Delta V_{1}$ suivi d'une phase à évolution plus lente, d'abord croissante puis décroissante (Fig. 2).

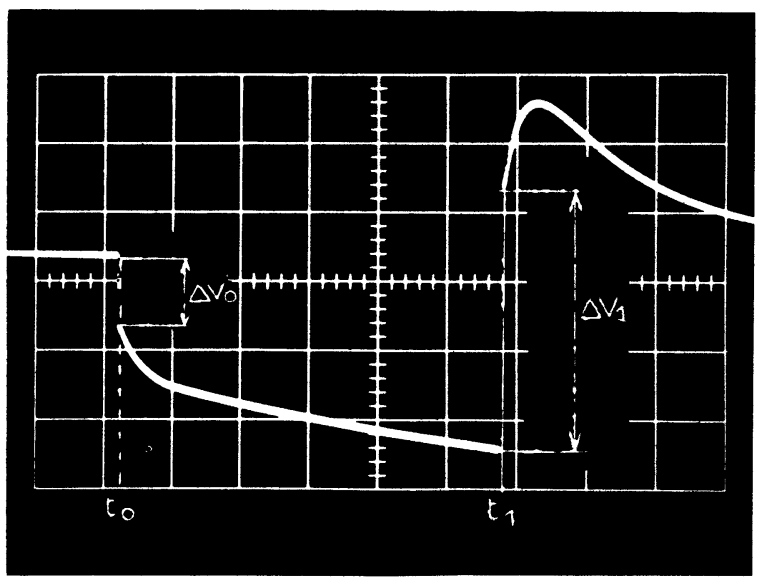

Fig. 2. - Photo de la tension aux bornes de la diode. courant appliqué :

$$
\left\{\begin{array}{rl}
t<t_{0} & I=140 \mathrm{~mA} / \mathrm{cm}^{2} \\
t_{0}<t<t_{1} & I=0 \\
t>t_{1} & I=140 \mathrm{~mA} / \mathrm{cm}^{2} .
\end{array}\right.
$$

De la même façon qu'au paragraphe précédent, nous pouvons écrire les deux sauts de tension $\Delta V_{0}$, $\Delta V_{1}$ :

$$
\begin{aligned}
& \Delta V_{0}=J_{0}\left[\int_{\mathrm{zc}} \frac{\mathrm{d} x}{\sigma_{0}(x)}+\int_{\mathrm{zd}} \frac{\mathrm{d} x}{\sigma_{0}(x)}\right] \\
& \Delta V_{1}=J_{0}\left[\int_{\mathrm{zc}} \frac{\mathrm{d} x}{\sigma_{\Delta t}(x)}+\int_{\mathrm{zd}} \frac{\mathrm{d} x}{\sigma_{\Delta t}(x)}\right]
\end{aligned}
$$

où $\sigma_{0}(x)$ est la conductivité stationnaire correspondant au courant $J_{0}$ et où $\sigma_{\Delta t}(x)$ est la conductivité restante au temps $t_{1}$.

Il est possible de déduire la durée de vie des porteurs de ces équations à condition de supposer $a$ ) que les chutes de tension dans les zones fortement dopées sont faibles par rapport à celle dans la zone centrale, b) que la décroissance de la densité de porteurs est exponentielle de sorte que

$$
\sigma_{\Delta t}(x)=\sigma_{0}(x) \exp \left[-\frac{\Delta t}{\tau}\right] .
$$

Avec ces hypothèses, on déduit la durée de vie dans la zone centrale :

$$
\frac{\Delta V_{0}}{\Delta V_{1}}=\frac{\int_{\mathrm{zc}} \frac{J_{0}}{\sigma_{0}(x)} \mathrm{d} x}{\int_{\mathrm{zc}} \frac{J_{0}}{\sigma_{\Delta t}(x)} \mathrm{d} x}=\mathrm{e}^{\Delta t / \tau}
$$

soit

$$
\tau=\frac{t_{1}-t_{0}}{\log \frac{\Delta V_{1}}{\Delta V_{0}}}
$$

On voit que cette méthode de mesure ne fait appel à aucun modèle de la jonction. La durée de vie ainsi mesurée est de plus peu sensible aux processus de disparition de porteurs qui interviennent sur les bords de la zone centrale (diffusion vers le centre ou extraction par les jonctions) : cela tient au fait qu'une variation de conductibilité dans ces régions où elle est déjà particulièrement élevée n'a qu'une incidence faible sur les intégrales de la formule (7). On peut donc s'attendre à ce que cette méthode de double impulsion donne la durée de vie des porteurs au centre de la structure, caractéristique de la recombinaison dans cette zone.

Enfin, l'évolution ultérieure de la tension aux bornes de la diode sous l'effet de la seconde impulsion de courant est caractérisée par la présence d'un maximum. L'injection de porteurs (ceux-ci augmentent en densité jusqu'à ce que le nouvel équilibre stationnaire soit atteint) est la cause de cet effet : l'accroissement de densité des porteurs a pour conséquence, d'une part l'augmentation de la tension de jonction, d'autre part la diminution de la chute ohmique de tension dans la zone centrale parcourue par un courant constant ; l'ensemble se traduit par un maximum de la tension appliquée observable avant que l'équilibre stationnaire soit atteint.

$b$. RÉSUltATS EXPÉRIMENTAUX. - Nous avons effectué une série de mesures sur des diodes au silicium $\mathrm{P}^{+} \mathrm{PN}^{+}$dont la zone intermédiaire avait une épaisseur de $500 \mu$ et une résistivité de $50 \Omega \mathrm{cm}$.

La figure 3 représente le schéma de principe du circuit de mesure. Le figure 2 montre la forme de la tension aux bornes de la structure au cours des deux 


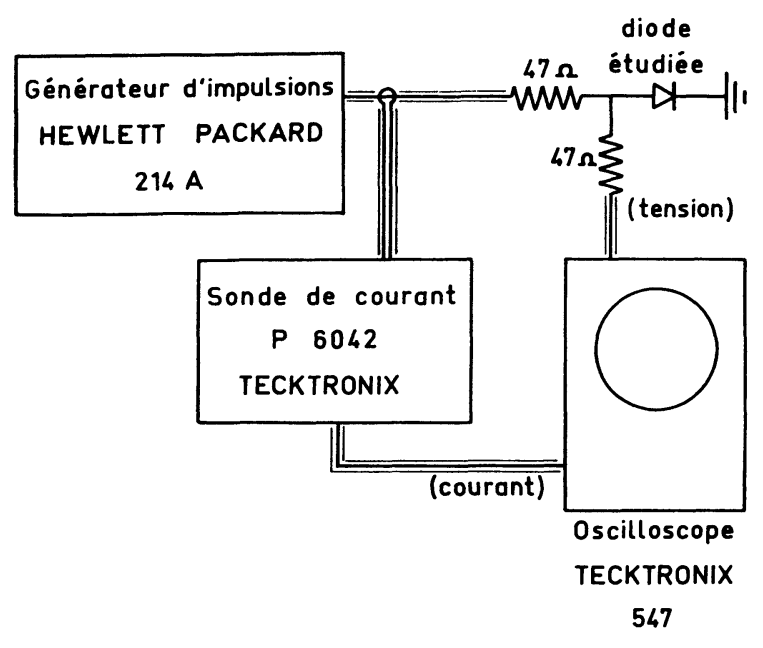

(tiroir 1 A 1)

FIG. 3. - Circuit de mesure.

impulsions successives de courant. Sur la figure 4, nous avons vérifié que la courbe

$$
\log \frac{\Delta V_{1}}{\Delta V_{0}}=\mathrm{f}(\Delta t)
$$

est bien une droite : cela traduit aussi une décroissance exponentielle de la densité des porteurs. Les durées de vie déduites sont portées sur la figure 5 en fonction de l'injection dans la diode. On peut comparer aux résultats obtenus par les deux méthodes classiques du temps de retournement et de la décroissance de la tension. L'indépendance de la durée de vie (mesurée par la méthode de la double impulsion) et du niveau d'injection est remarquable. Ce résultat s'accorde avec l'idée d'un processus unique de disparition des porteurs, à savoir la recombinaison, au centre de la structure. De même la valeur élevée de cette durée de

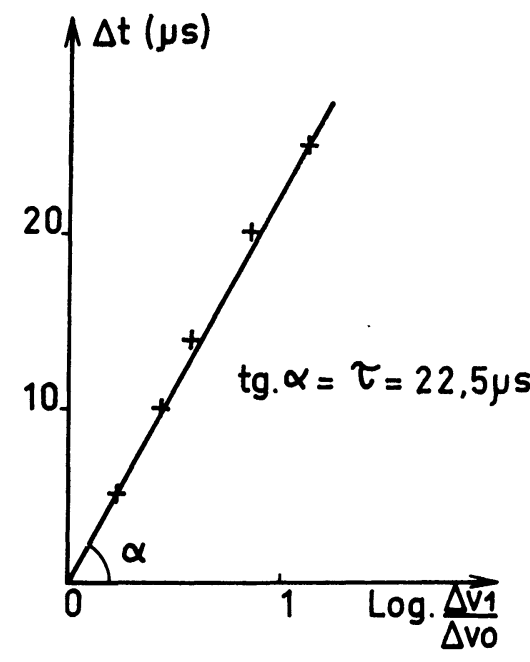

FIG. 4. - Courbe $\quad \log \frac{\Delta V_{1}}{\Delta V_{0}}=\mathrm{f}(t)$.

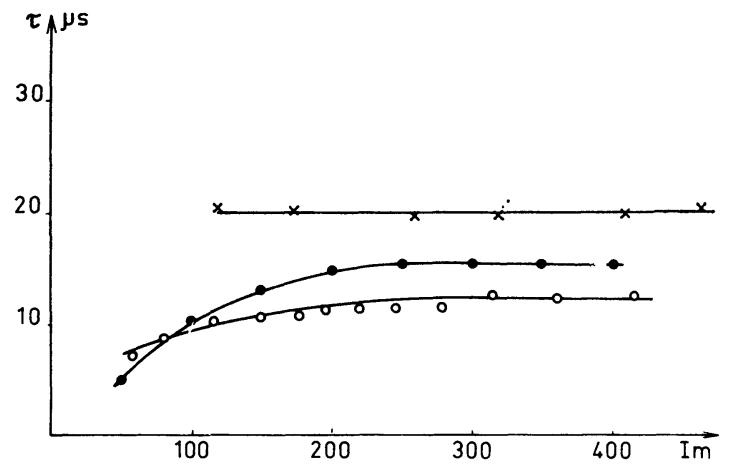

Fig. 5. - Durée de vie en fonction du courant direct $(\times$ méthode de la double impulsion; $\bullet$ temps de retournement; $\circ$ décroissance de la tension en circuit ouvert).

vie peut être attribuée à une faible contribution des extrémités dans la disparition des porteurs au centre de la structure.

\section{Bibliographie}

[1] Lederhandler (S. R.) et Giacoletto (J. L.), Proc. Instu. Radio Engrs, 1955, 43, 477.

[2] Lax (B.), et Neustadter (S.), J. Appl. Phys., 1957, $28,8$.

[3] Hoffmann (A.) et Schuster (K.), Solid St. electron., $1964,7,717$.

[4] Davies (L. W.), Proc. I. E. E. E., 1963, 51, 1637.

[5] Wilson (P. G.), Solid State electron., 1967, 10, 145.

[6] Schangenotto (H.) et Gerlach (W.), Solid st. electron., 1969, 12, 267.
[7] BASSETt (R. J.), Solid St. electron., 1969, 12, 385.

[8] Brousseau (M.) et Schuttler (R.), C. R. Acad. Sci. Paris, 1968, 266, 502.

[9] Brousseau (M.) et SChuttler (R.), Solid St. electron. 1969, $12,417$.

[10] Barsukov (Yu. K.), Soviet Phys. Tech. Phys., 1958, 806.

[11] Baron, J. Appl. Phys., 1965, 37, 7, 2614

[12] Shoo (S. C.) et MAzUR (R. G.), Solid St. electron., 1970, 13, 553. 